\title{
Capitate Osteonecrosis: A Pediatric Case Report
}

\author{
Davod Jafari, ${ }^{1}$ Hooman Shariatzadeh, ${ }^{1}$ and Razieh Nabi ${ }^{1,}$ \\ ${ }^{1}$ Bone and Joint Reconstruction Research Center, Shafa Orthopedic Hospital, Iran University of Medical Sciences, Tehran, Iran \\ "Corresponding author: Razieh Nabi, Bone and Joint Reconstruction Research Center, Shafa Orthopedic Hospital, Iran University of Medical Sciences, Tehran, Iran. Tel: \\ +98-9124847086, Fax: +98-2133542020, E-mail: md_natan@yahoo.com
}

Received 2016 December 23; Revised 2017 February 27; Accepted 2017 March 10.

\begin{abstract}
Introduction: Avascular necrosis (AVN) of the capitate is relatively rare. Although there are many factors as etiology; however, there are idiopathic ones.

Case Presentation: A15-year-old female presented with wrist pain without the history of previous major trauma and no relief with conservative management; radiographic evaluation revealed capitates osteonecrosis with collapse and sclerosis. She underwent surgery (curettage of necrotic bone and iliac crest bone grafting). Two years fallow-up showed full recovery clinically and radiographically.

Conclusions: Capitate AVN should be included in the differential diagnosis of wrist pain in pediatric patients. Despite the controversial multiple surgical options to treat capitate osteonecrosis, autogenous iliac crest bone grafting can have a good result, even in the pediatric patient.
\end{abstract}

Keywords: Capitate Bone, Osteonecrosis, Pediatric

\section{Introduction}

Idiopathic avascular necrosis (AVN) of the capitate is a rare condition that usually increases in the late teens or early $20 s$ (1). Most of the cases are accompanied by serious trauma, but in some cases, only a few reports of idiopathic capitate AVN are published (2). Several surgical treatments are described in the literature, including interposition arthroplasty using tendon graft, intercarpal arthrodesis, silicone arthroplasty, resection of the proximal pole and drilling, and free vascularized bone graft from iliac crest (1). The current report is on non-vascularized bone graft from iliac crest as a treatment for this rare condition; resulting in full recovery clinically and radiographically at 2year fallow-up.

\section{Case Presentation}

The case was a 15-year-old, right-handed female presented with a 3-year history of wrist pain and mild swelling in her right wrist. The pain was predominantly located in the middle of the dorsal wrist and was aggravated by wrist motion. She also mentioned stiffness and significant weakness of the right wrist. There was no history of previous wrist trauma. She had received conservative treatment including nonsteroidal anti-inflammatory medicine and splinting by her primary orthopedist, which failed to reduce her symptoms. At her 1st visit to Shafa hospital, slight dorsal swelling of the right wrist was noted. There was moderate tenderness in palpation over the dorsum of the right wrist centrally. The range of wrist motion from right to left was subsequently: dorsiflexion, $70^{\circ}-90^{\circ}$; and palmar flexion, $60^{\circ}-90^{\circ}$. Radiographs and computed tomography showed cystic changes of the capitate with slight collapse of its proximal pole (Figure 1); in addition, there was an incidental finding, luno-triquetral coalition. T1-weighed magnetic resonance imaging (MRI) showed an area of heterogeneous low signal intensity in the entire capitate (Figure 2). $\quad A_{99 m}$ Tc-methylene diphosphonateenhanced scintigraphy revealed a focus of increased uptake in the carpus (Figure 3). Because of the patient's pain and weakness, surgical treatment was recommended. The procedure was done under general anesthesia with tourniquet control. A5-cm longitudinal incision was made on the dorsal wrist. The extensor retinaculum was, then, identified and reflected to expose the 4 th and 5 th extensor compartments. The capsule and, then, carpal bones were exposed through these 2 compartments. In the next step, curettage of the capitate and autogenous grafting of the corticocancellous iliac bone were conducted. The wrist was immobilized in short arm cast for 6 weeks and therapy was started afterward to improve the range of motion of joints. It was observed that the medullary bone of the capitate was pale yellow and sclerotic. The specimen was sent to the pathologic examination, the result was combination of necrosis and regeneration of the trabecular bone. After surgery, a significant improvement in subjective symptoms and physical findings was observed. Two years after 
surgery, the patient had full range of motion of the wrist, and also she did not have any pain. Radiographs showed solid bone union of the capitate and preservation of its proximal articular surface (Figure 4). The parents of the patient signed written consent to publish the report and the study was approved by the ethic board of Shafa orthopedic center.
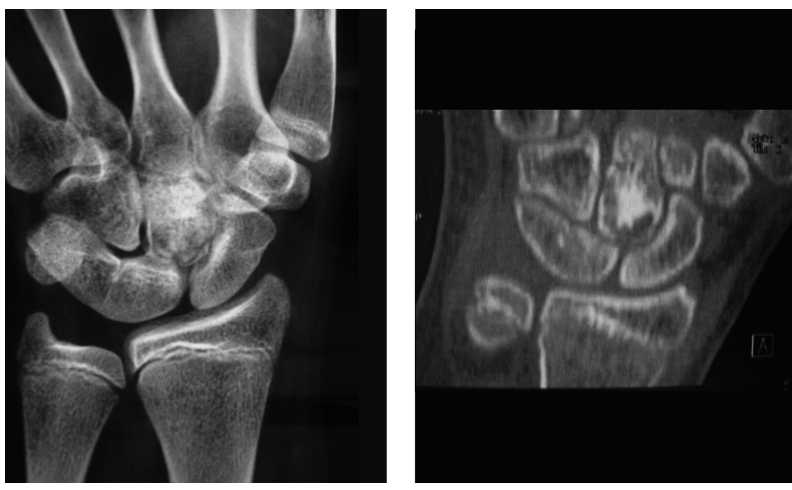

Figure 1. Radiograph and computed tomography scan showing increased density in capitate corresponding to the zone of avascular necrosis. Luno-triquetral coalition is present as incidental finding.

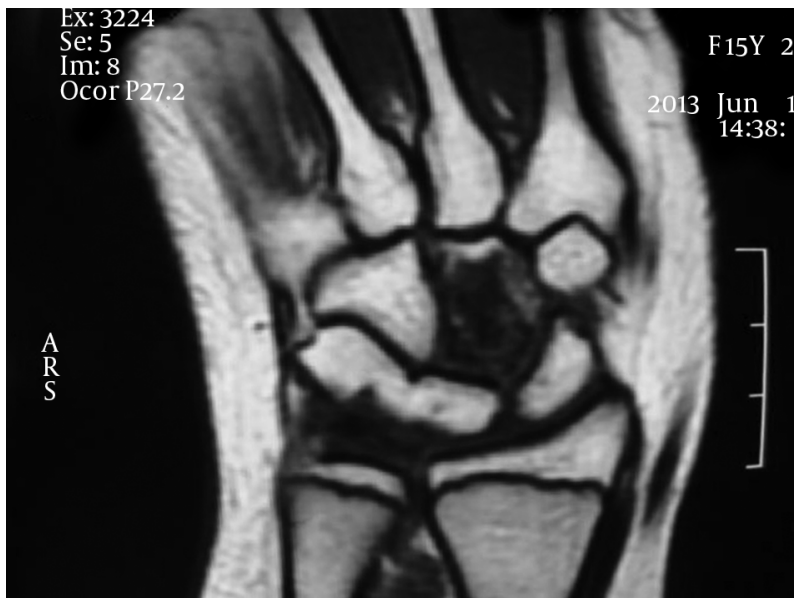

Figure 2. T1-Weighed MRI Showing an Area of Heterogeneous Low Signal Intensity in the Entire Capitate

\section{Discussion}

AVN of the capitates is a rare clinical entity, 1st reported by Jonsson in 1942 (3), and notably, it is more rare in pediatric population as in the current case; in the literature there were only 2 cases younger (5- and 14-year-old males) than the patient in the current study (4). More than half

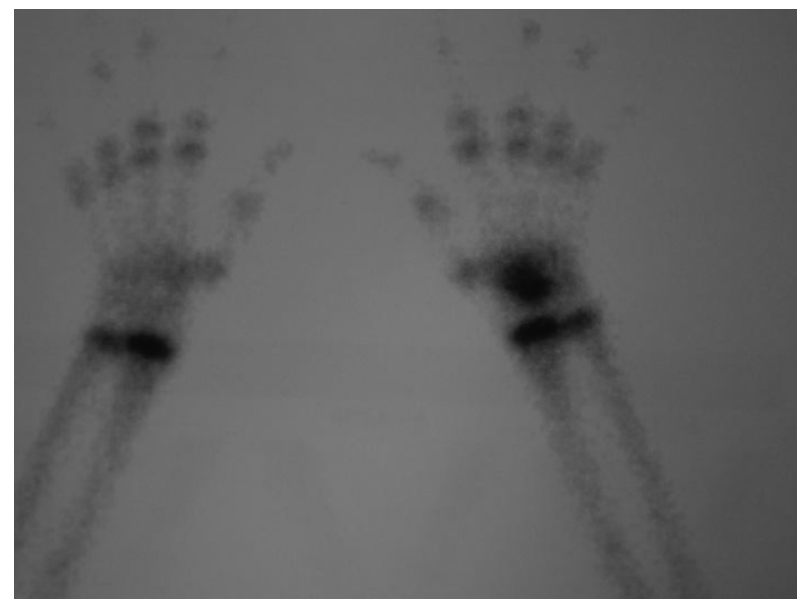

Figure 3. Increased Uptake Represents Revascularization and Osteoblastic Activity

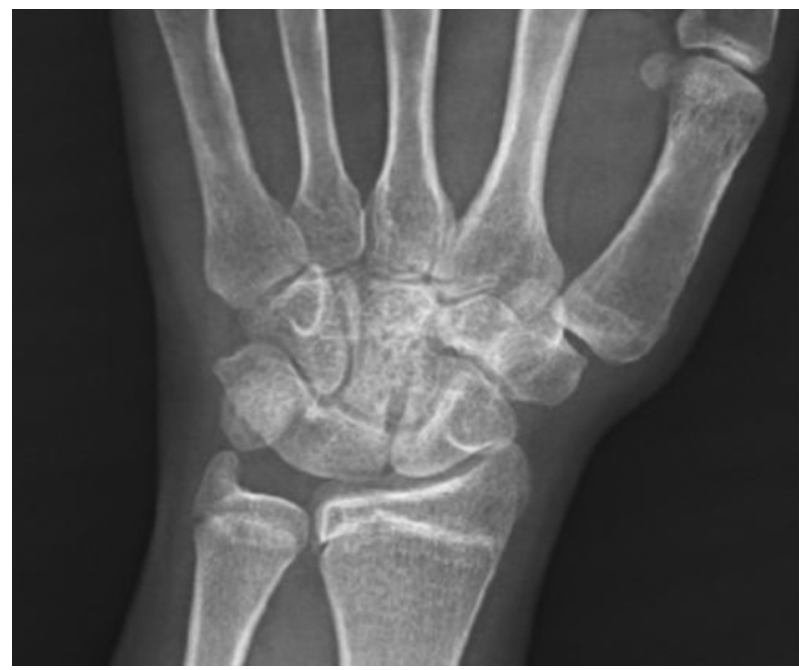

Figure 4. Two Years After Surgery, Radiograph Showing Solid Bone Union of the Capitate

of the reported cases had a history of trauma because of repetitive motion. Local steroid injection is also thought to cause AVN of the capitate bone (5); but none of them were present in the current case report and it seems to be the idiopathic one. The vascular supply to the proximal pole of the capitate is tenuous, and analogous to the vascular supply of the scaphoid (6). The head of the capitate has no collaterals; thus, it is at further risk for AVN, but infrequency of AVN of the capitate, compared with that of the scaphoid, is thought to be related to its relatively protected position in the center of the distal carpal row. Low signal intensity on T1-weighted images is the most reliable indicator of the presence of AVN (7); compatible with the MRI 
finding of the current study patient. Murakumi et al., reported MR imaging of a case of AVN of the capitate with evidence of low-signal intensity areas on T1-weighted images and high-signal intensity areas on T2 images, corresponding to an area of revascularization and regeneration noted on angiography and histologic examination (5). Bekele et al., introduced a case with the MRI finding as follows: the proximal pole of the capitate demonstrated extremely lowsignal intensity on both T1- and T2-weighted images of the wrist. By all known standards and grading systems, this corresponds to a late stage of AVN. More heterogeneous signal intensity within the distal pole is of less certain significance, the possibilities include moderate to severe ischemia versus revascularization or reparative process (7). Often AVN is diagnosed using the 3-phase skeletal scintigraphy. Initial presentation of AVN is obvious reduced vascularity in all the 3-phase, but over the time, due to osteoblastic activity in necrotic bone, vascularity would increase, which enhance uptake subsequently (8). The current study patient referred after prolong symptoms, it was expected that skeletal scintigraphy would represent enhanced uptake due to revascularization. Mansberg et al., showed that scintigraphic features were different, based on the time in early onset stage; significantly reduction in vascularity in the proximal carpal row of the hand in both phases blood flow and pool were observed with no uptake in the delayed images throughout the midcarpus, but when the bone scan was repeated 3 months later, vascularity and osteoblastic activity in the capitate bone increased (8). The histological findings (necrosis and regeneration of the trabeculae) also supported the imaging findings that blood supply had resumed. Surgical intervention is usually warranted for AVN of the capitate.

For the current study patient, curettage of the capitate bone marrow and also autogenous grafting of the corticocancellous iliac bone were done. Other surgical procedures included resection (6), arthrodesis (5), drilling (9), arthroplasty (10), and vascularized pedicle bone grafting (1). There are numerous studies about surgical procedures to reduce pain and improve structural integrity. However, the useful method to improve wrist function is still obscure because it is really uncommon. When a lesion is found in early stages of necrosis, immobilization of the wrist should be used as a reasonable treatment. In conclusion, capitate AVN should be diagnosed differently from chronic wrist pain and curettage, and bone graft can result in recovery in clinical and radiographic findings.

\section{Footnote}

Authors' Contribution: Davod Jafari, Hooman Shariatzadeh and Razieh Nabi: all were involved in diagnosis, treatment of the patient, and in drafting and revising of the manuscript.

\section{References}

1. Hattori Y, Doi K, Sakamoto S, Yukata K, Shafi M, Akhundov K. Vascularized pedicled bone graft for avascular necrosis of the capitate: case report. J Hand Surg Am. 2009;34(7):1303-7. doi: 10.1016/j.jhsa.2009.04.012. [PubMed: 19497683].

2. Ichchou L, Amine B, Hajjaj-Hassouni N. Idiopathic avascular necrosis of the capitate bone: a new case report. Clin Rheumatol. 2008;27Suppl 2:S47-50. doi: 10.1007/s10067-008-0865-5. [PubMed:18345477].

3. Kutty S, Curtin J. Idiopathic avascular necrosis of the capitate.J Hand Surg Br. 1995;20(3):402-4. [PubMed: 7561422].

4. Humphrey CS, Izadi KD, Esposito PW. Case reports: osteonecrosis of the capitate: a pediatric case report. Clin Orthop Relat Res. 2006;447:256-9. doi: 10.1097/01.blo.0000203459.12759.29. [PubMed: 16505704].

5. Murakami H, Nishida J, Ehara S, Furumachi K, Shimamura T. Revascularization of avascular necrosis of the capitate bone. AJR Am J Roentgenol. 2002;179(3):664-6. doi: 10.2214/ajr.179.3.1790664. [PubMed: 12185039].

6. Vander Grend R, Dell PC, Glowczewskie F, Leslie B, Ruby LK. Intraosseous blood supply of the capitate and its correlation with aseptic necrosis. J Hand Surg Am. 1984;9(5):677-83. [PubMed: 6386955].

7. Bekele W, Escobedo E, Allen R. Avascular necrosis of the capitate. J Radiol Case Rep. 2011;5(6):31-6. doi: 10.3941/jrcr.v5i6.760. [PubMed: 22470799].

8. Mansberg R, Lewis G, Kirsh G. Avascular necrosis and fracture of the capitate bone: unusual scintigraphic features. Clin Nucl Med. 2000;25(5):372-3. [PubMed: 10795699].

9. DeSantis DP. Postsurgical rehabilitative management of avascular necrosis in the capitate. J Manipulative Physiol Ther. 2004;27(8):519-24. doi: 10.1016/j.jmpt.2004.08.006. [PubMed: 15510096].

10. Bolton-Maggs BG, Helal BH, Revell PA. Bilateral avascular necrosis of the capitate. A case report and a review of the literature.J Bone Joint Surg Br. 1984;66(4):557-9. [PubMed: 6746692]. 